\title{
An Enhanced Method for Detecting the Shaded Images of the Car License Plates based on Histogram Equalization and Probabilities
}

\author{
Mohammad Faghedi ${ }^{1}$, Behrang Barekatain ${ }^{2} *$ \\ Computer Engineering Department \\ Najafabad Branch, Islamic Azad University \\ Najafabad, Iran
}

\author{
Kaamran Raahemifar ${ }^{3}$ \\ Electrical \& Computer Engineering Department \\ Ryerson University \\ Toronto, Ontario, Canada
}

\begin{abstract}
Shadow is one of the major and significant challenges in detection algorithms which track the objects such as the license plates. The quality of images captured by cameras is influenced by weather conditions, low ambient light and low resolution of the camera. The shadow in images reduces the reliability of the sight algorithms of the device as well as the visual quality of images. The previous papers indicate that no effective method has been presented to improve the license plate detection accuracy of the shaded images. In other words, the methods that have been presented for automatic license plate detection in shadowed images until now use a combination of color features and texture of the image. In all these methods, in order to detect the frame of the shadow and the texture of the image, sufficient light is required in the image; this necessity cannot be found in most of the regular images captured by road cameras. In order to solve this problem, an improved license plate detection method is presented in this research which is able to detect the license plate area in shadowed images effectively. In fact, this is a contrast-improving method which utilizes the dual binary method for automatic plate detection and is introduced to analyze the interior images with low contrast, and also night shots, blurred and shadowed images. In this method, the histogram of the image is firstly calculated for each dimension and then the probability of each pixel in the whole image is obtained. As a result, after calculating the cumulative distribution of the pixels and replacing it in the image, it will be possible to remove the shadow from the image easily. This new method of detection was tested and simulated for 1000 images of vehicles under different conditions. The results indicated the detection accuracy of $90 / 30,97 / 87$ and $98 / 70$ percent for the license plates detection in three databases of University of Zagreb, Numberplates.com and National Technical University of Athens, respectively. In other words, comparing the performance of the proposed method with two similar and new methods, namely Hommos and Azam, indicates an average improvement of $26 / 70$ and $72 / 95$ percent for the plate detection and $32 / 38$ and $36 / 53$ percent for the time required for rapid and correct license plate detection, even in shaded images.
\end{abstract}

Keywords-Automatic license plate detection; shadowed images; histogram equalization; cumulative distribution; pixel probability

\section{INTRODUCTION}

License plate detection for vehicles is a frequently used technique in the field of image processing. The key role of the license plate detection is controlling as well as managing the implementation of traffic rules automatically in public roads. Each vehicle carries a unique license plate; therefore, no external card, label or transmitter is needed. Furthermore, license plates can be used for traffic management, border control, access control and parking cost. Before this, general technologies such as Vehicle Identification Number (VIN) and electronic labels were used to identify the vehicles, which were quite expensive. On the other hand, if a new vehicle was produced, one of such technologies should have been installed on it in order to enable us to identify such vehicles. To remove this factor, the best method to identify a vehicle is detecting the car license plate identification number, utilizing a simple camera. One of the most important steps for identifying the license plate number of vehicles is detecting the license plate area and extracting it from the image of the vehicle. Most of the license plate detection software deal with challenges while extracting the license plate area from the image; among these challenges, the followings can be addressed: Existence of various types of texts around the license plate, existence of unwanted objects in the image, low ambient light, presence of more than one vehicle in a single image, deviation of the license plate and existence of a shadow fallen on the license plate. Shadow detection is one of the important aspects of most of the detection and object tracking algorithms. Shadows appear in an image when objects block the light emitted from the source of light; therefore, they appear as the surface features. The procedure of shadows detection is divided into three processes: low level, mid-level and high level [1]. The low level process detects the areas that are darker than their surroundings. Shadows are among the dark areas. The midlevel process detects the features among the dark areas such as penumbra and shadow. The location of the object is beside the dark areas. A high level process then merges such assumptions and verifications of stabilities among the light directions estimated from them. The shadow area, generally, is divided into two sections: the shadow itself and the shadow frame. The shadow itself is a part of an object that does not appear by direct light. The shadow frame is the area that appears by the object on the direct path of light. The shadow frame, in the real world, is dependent on the effects of light, as the light beam encounters more than one reflection from the object's surface in its path from the light source. The dark spot is a part of the shadow frame that is completely blocked by the 
object itself. On the other hand, penumbra is a part of the shadow frame that has blocked the direct light in a scattered manner. These parts are described in Figure 1:

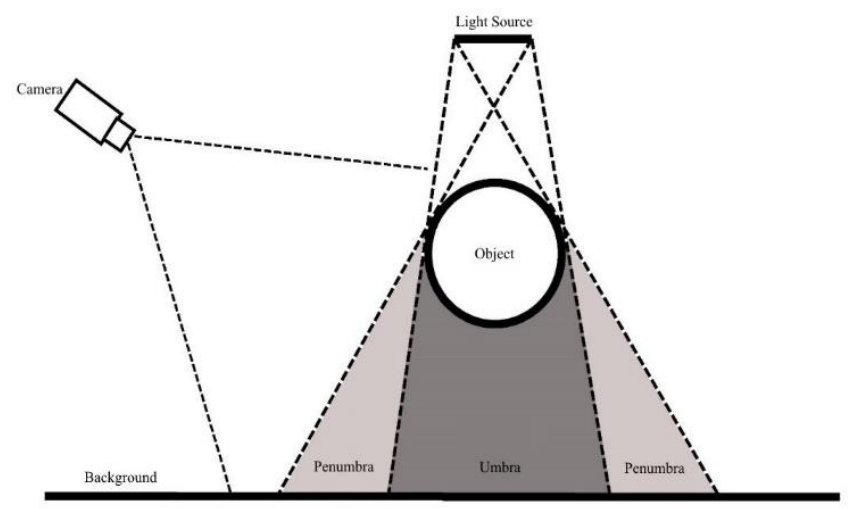

Fig. 1. Cast shadow parts : "umbra" and "penumbra" [2]

A spot light source only causes the appearance of a dark spot in the shadow. An area of light source, however, causes the appearance of both a dark spot and penumbra. When the penumbra is very small, it might be detectable considering the effect of digitalizing the image. A dark spot has a low intensity of brightness due to the fact that it does not receive any light from the light source. Such intensity increases gradually from the dark spot to the penumbra. Shadows might be considered as a part of the object's backgrounds; in this case, they could lead to the merge of the backgrounds, deviation of the objects' shape and even their disappearance (considering that the shadow frame has fallen on the other objects as well). Therefore, removing the shadows leads to more accurate detection of the vehicle and consequently, a more accurate background for the license plate algorithms. Several methods have been presented until now to detect the shadows and remove them from the license plate image, including utilization of the Bernsen algorithm [3, 4], utilization of the color features of the license plate [5], the counting algorithm $[6,7]$, utilization of the Otsu's threshold $[8,9]$, detection of the vertical and horizontal edges $[10,11]$ and utilization of the edge density in order to increase the contrast of the image $[12,13]$. All the presented methods are very sensitive to the noise and if the shadow has covered a major part of the license plate and the ambient light is low, these methods face problems. In this research, an improved method is presented to remove the shadow on the license plate and to detect the license plate area. For this purpose, first, the histogram of the image is calculated for each dimension; then after obtaining the cumulative distribution of the pixels and replacing them with the obtained number in the image, we can remove the shadows from the images; then utilizing Tamura contrast improvement, image quality is improved and therefore, we proceed with effective detection of the license plate area within the image. The proposed method has been tested on 1000 images of vehicles under different conditions and has obtained the average performance of 95/62 percent for detecting license plates in all three databases. Also the performance of the proposed automatic license plate detection method was compared with two new Hommos [14] and Azam [15] methods. The proposed automatic license plate detection method provides an average improvement of 20/13 percent for the accuracy of the license plate detection rate and an average improvement of $32 / 38$ percent for the implementation time, compared to other methods. The structure of this article is categorized as following: In the second section, we will address previous works and then the problems of the previous methods are categorized in the third section. In the fourth section, the proposed method is described with necessary details, and then evaluation and analysis of the results are provided in the fifth section and finally, in the sixth section, the article is concluded.

\section{RELATED WORK}

Since previous decades, there have been several attempts to solve the problem of detecting the license plate area from the image. Various advanced methods, various image processing methods, techniques and algorithms have been utilized for establishing automatic license plate detection methods. Several features such as geometric, texture and color features were either separately or jointly used in order to detect the license plate area. Figure 2 demonstrates the categories of the presented methods for license plate detection. Some of the advanced automatic license plate detection methods and their limitations are summarized and described in the following:

Anagnostopoulos et al. [16] have presented a license plate detection system. The automatic license plate detection phase of this system receives a gray image as the input and utilizes a segmentation technique, called Sliding Concentric Windows (SCW), for selecting the rich texture area in the image. In the analysis of connected components, the parts that are not license plates are rejected based on the surface area, direction and calculation of the Euler number. In the license plate area, however, the Euler number is very sensitive to small noises. To remove the noises, no method is presented in the automatic license plate detection method. This automatic license plate detection method has not considered the effects of the weather conditions, license plate deviation and low contrast of the image and makes no specific effort in order to solve these issues. Kaushik et al. [17] have utilized an improved version of the Sliding Concentric Windows (SCW) that detects the vertical and horizontal edges in order to detect the license plate area. After that, only the rectangular shaped areas are kept on the edge image based on the shape and size of the license plate. In the end, the connected components are extracted from the image and all those that are not license plates are filtered based on the surface area and color information. One of the limitations of this method is the utilization of the rectangular information of the license plate edges for the initial detection, because the detection of the concerned license plate is not always possible from the edge information due to the low contrast between the license plate and the body of the automobile. Furthermore, this automatic license plate detection method has not considered the effects of weather conditions, low contrast and license plate deviation. Wang et al. [18] have presented an automatic license plate detection method based on the Discrete Wavelet Transform (DWT) and the Sliding Concentric Windows. The automatic license plate detection method fails when the edges are unclear due to the low contrast of the image. This 
automatic license plate detection method contains no phases for analyzing unnecessary edges in various weather conditions and also, there is no specific phase in that for analyzing the horizontal deviation of the license plate and noises. In addition, to locate the license plate, this method only utilizes the edge density; therefore, it is not an appropriate method for detecting the license plates in high-risk situations. Ashim et al. [19] have presented a license plate identification method for the license plates written in Bengali language. The automatic license plate detection phase of this system takes the gray image as the input and utilizes the medium filter in order to remove the noise. Then Sobel vertical operator is applied for detecting the vertical edges of the image. Thresholding is used to extract the candidate areas as license plates and select some rows based on the density of edges. Morphological erosion and dilation are applied to the selected edges in order to remove unwanted objects. In the end, the main license plate area is obtained using the vertical cropping based on the density of edges. To remove the areas that are not license plates, the surface area is utilized only as the main filter. There is no concern about the issue of the effects of weather conditions and license plate deviation in this automatic license plate detection method. This method is not even concerned with analyzing the night shots, blurred and shadowed images. The medium filter is utilized in the initial phase in order to remove the noise which indicates a questionable performance. Another limitation is using the edge density and the amount of threshold (histogram analysis) for horizontal cropping of the image, since an image may include other objects with high edge density; therefore, when several objects overlap with each other, a huge error happens in horizontal cropping. Using the analysis of the connected components instead of histogram analysis can be considered as a better method.

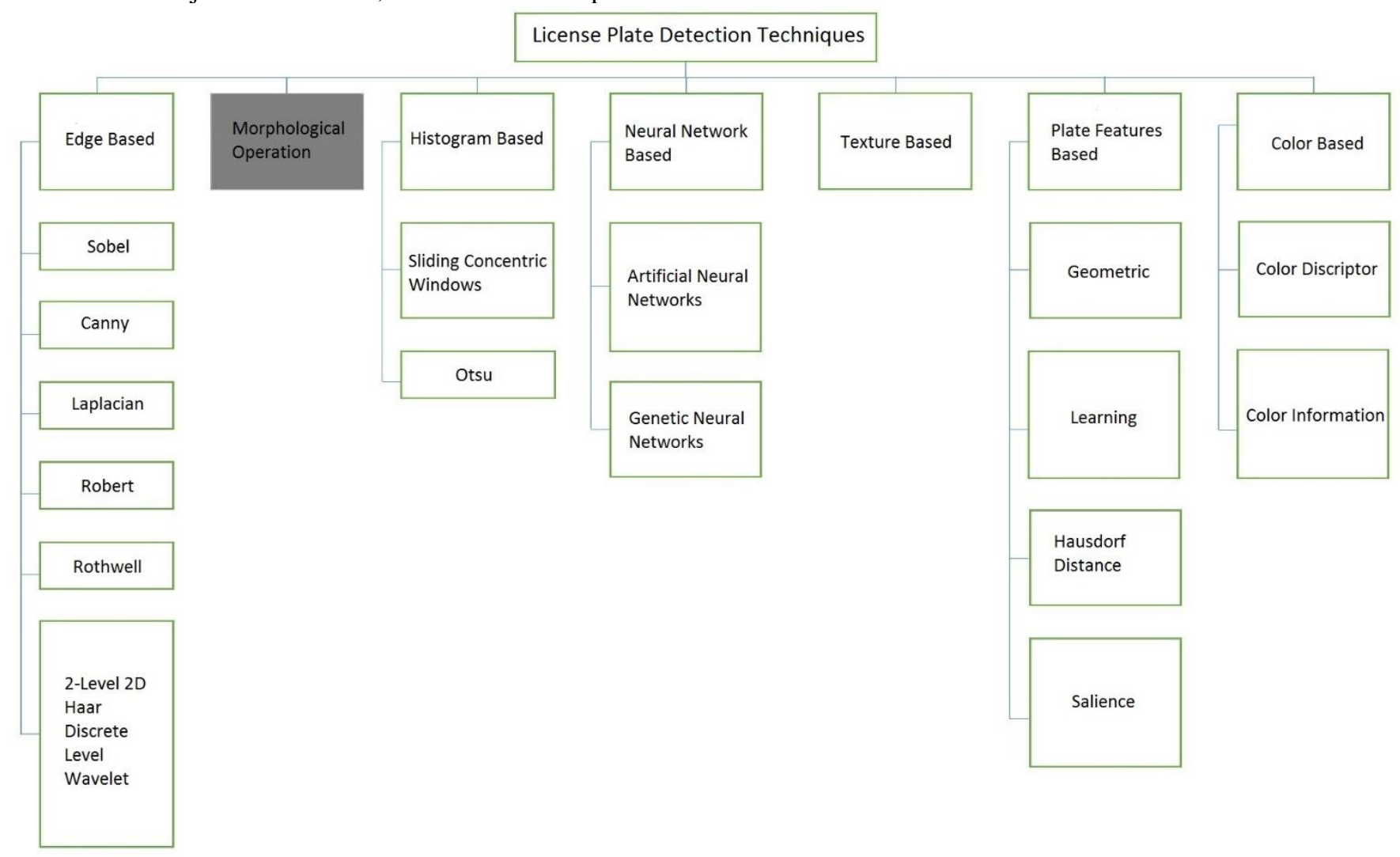

Fig. 2. Classification diagram

Joarder et al. [20] have presented another license plate detection method based on the density of the vertical edge. This automatic license plate detection method uses the density of the edge in order to increase the local contrast. Therefore, the contrast of dense areas increases compared to the low density areas. Nonetheless, this method fails when the edge information is weak in the license plate (night shots or blurred shots should be considered). Another problem is that before applying the contrast improvement method, no noise removing filter is used. Hsu et al. [21] have presented a softwareoriented automatic license plate detection method whose parameters can be set in order to be placed in the system for three different programs: access control, rules implementation and road patrol. This method utilizes vertical edge clustering method for detecting dense areas of the edge in the image, in automatic license plate detection phase. Eventually, the areas that are not license plates are rejected based on the surface area, size and direction. This automatic license plate detection method has no techniques for removing the rain drops and improving the contrast of the image. Further, rain drops increase unrelated edges and the low contrast of the image also decreases the detection of sharp edges in the image; this misleads the clustering method and reduces the detection performance of the license plate areas. Nejati et al. [22] have 
presented a license plate detection system. Their proposed algorithm consists of four main steps including: license plate positioning, segmentation, identification and a combination of the results of multiple identifications. License plate positioning commences with some preprocessing, low sampling, removing the Gaussian noise and dynamic setting of the light intensity range with histogram equalization. Then the histogram of the vertical edges is used to detect the concerned candidate lines including the license plate. Issues such as weather conditions, blurred and shadowed images are not solved in this method. Azam et al. [15] have presented an automatic license plate detection system for rainy weather conditions. In this system, the color image is converted to gray and then rain drops are removed from the image utilizing Fourier transformation discrete. Then Radon transform is used in order to solve the issue of horizontal deviation of the license plate. Whereas Bernsen method is very sensitive to the noise and is used in this research to binarize the image, it fails in conditions such as low contrast and existence of the shadow in the image. Hommos et al. [14] have presented an automatic license plate detection system. This system works on High Definition (HD) images. In this method, first, HD images are converted to Standard Definition (SD) images and then they are resized. In the next step, the color image is converted to the gray image. A series of morphological operations such as erosion and dilation are applied to the gray image and then the binary image is made, using a constant threshold. No special conditions, such as weather conditions, blurriness and existence of the shadow, existence of objects or texts in the image which are similar to the license plate, are considered in this method. Also, there is no particular method expressed in this method for removing the noise or the license plate deviation. Li et al. [23] have presented an automatic license plate detection system in order to detect the license plates of vehicles. In this method, inspired by the success of the Deep Neural Networks (DNNs) in various vision programs, the power of DNNs for learning high level features in a cascade framework is considered, which leads to improved performance both in detection and identification. In the beginning, a class of 37 convolutional neural networks is trained for detecting all characters in an image; compared to other common methods such as categorizing a binary text or a non-binary one, it leads to a high rate of calling. The incorrect items are then removed by the second categorizing of the license plates or non-license plates. Then the box area is refined in order to improve the connectedness intersection ratio based on the information of the license plate edge. The proposed cascade framework extracts the license plates effectively and with a high accuracy and recalling. In the end, after detecting the license plate characters, a method is presented under the title of comet labeling issue. A Recurrent Neural Networks (RNN) with Long Short-Term Memory (LSTM) is trained with a convolutional neural network to identify the sequential features extracted from the whole license plate. The major advantage of this method is its free segmentation. RNN method performs better than the basic segmentation combination and categorization of the deep convolutional neural network through analyzing the background information and preventing errors caused by segmentation; therefore, it reaches high identification accuracy according to the latest scientific advancements. The main focus of this method is on identifying the characters and reading the license plates. In the license plate detection phase, this method assumes that there is no special condition in the image; therefore, it does not consider the existence of the shadows in the images and does not provide any solutions for that either.

\section{PROBLEM STATEMENT}

As it was addressed previously, to detect the license plates in shadowed images, we face challenges. Previous methods indicate that this dilemma has not been considered accurately and efficiently. An effective method which is able of solving this problem should own the following items:

1) It should be resistant to the noise.

2) It should be resistant to the low ambient light and should be able to coordinate the ambient light.

3) It should be able to remove the shadows in a way that causes no harms to the texture or the details of the license plate.

4) It should be able to operate well on the color images too.

5) It should be able to extract the license plate from the image effectively in different conditions (day, night, blurred or shadowed images).

In the next part, we will present a new method for solving the above mentioned issues.

\section{MATERIALS AND METHOD}

Unlike Bernsen algorithm that is very sensitive to the noise, the proposed method here is noise resistant. After removing the shadow and improving the ambient light, it will be easy to utilize counting algorithms or vertical or horizontal edge detection algorithms. By utilizing the hysteresis threshold, unlike Otsu's method, it is possible to binarize the image with more details and also, by utilizing Tamura contrast improvement, it is possible to improve the quality of the image. These series of actions will ease the trend of detecting the license plate from the image and make it more accurate. In addition, not only is the proposed method noise resistant, it is resistant to color and gray images as well and therefore, it can be used for color images. Generalities and details of the proposed method are addressed in the following.

\section{Generalities of The Proposed Method}

Since 2010, new articles have been presented on license plate detection based on morphological and texture methods $[14,15,24,25]$. Although these methods could solve the problems of license plate detection to some extent, researches conducted in this field were not able to analyze the issues such as having shadows in the images appropriately; therefore, they lacked necessary and sufficient precision for detection. The main focus of this research is on improving the precision and accuracy of license plate detection in shadowed images. In the proposed method, histogram equalization is utilized based on the probabilities of both dimensions of the image to remove the shadows from the images and improve the contrast of the images as well. After noise is removed, using Wiener's 
method, the image is binarized, using Hysteresis threshold. Morphological operators are employed and finally, the connected components analysis method is utilized. To filter the areas that are not license plates, in addition to size and surface conditions, the value of Entropy (E) is taken into consideration in order to remove the items that are not license plates. Furthermore, in this research, the technique of histogram modification based on probabilities has solved the problems related to the detection of shadowed license plates in previous methods to a great extent; also, having considered the researches and having them compared with other presented methods, we have obtained a higher rate of accuracy and a shorter average performance time in the license plate detection. The proposed automatic license plate detection method has six stages that are demonstrated in Figure 3.

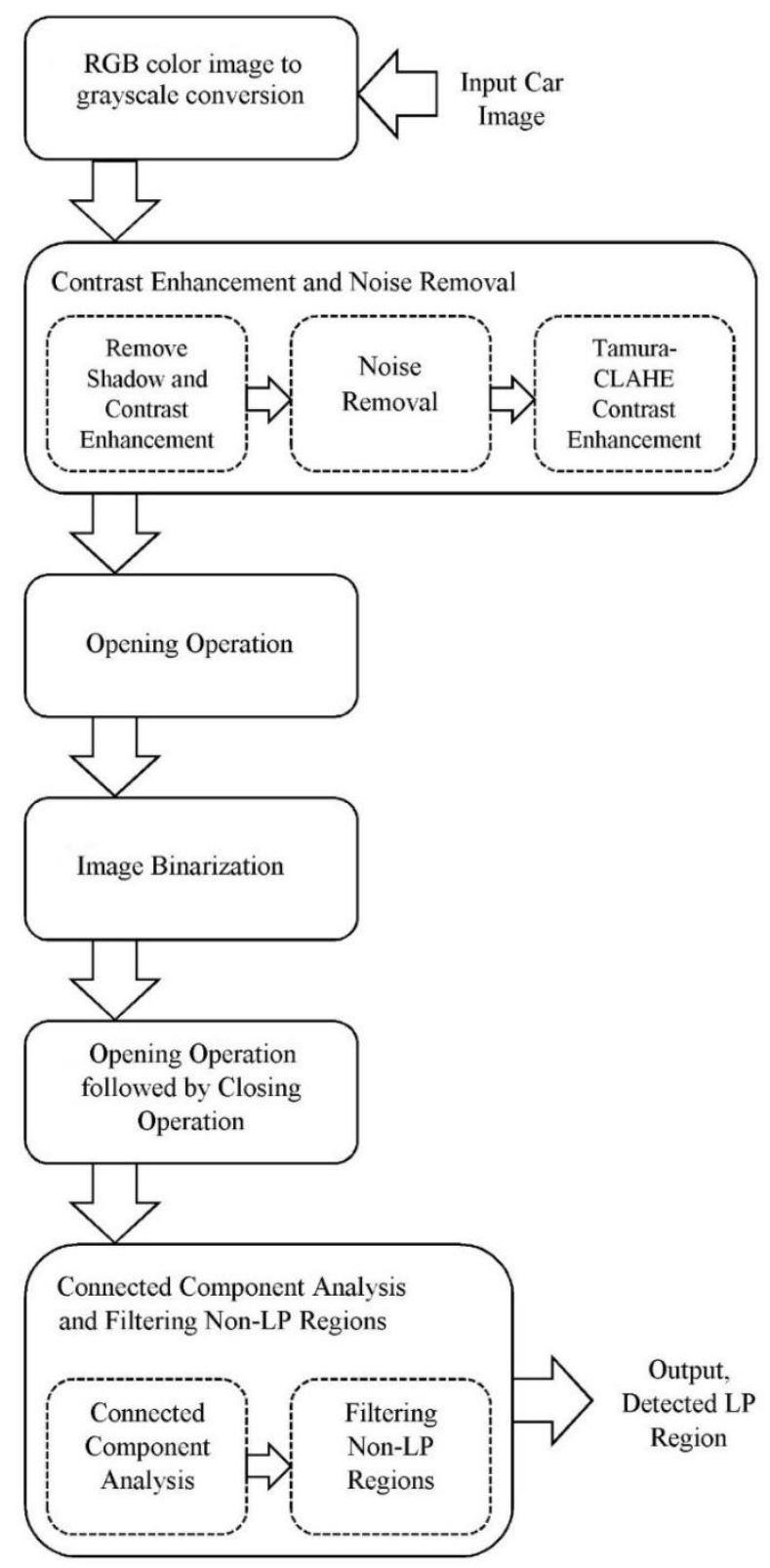

Fig. 3. Sequential stages of the proposed ALPD method

\section{Details OF The PROPOSED Method}

Our purpose is to present a new automatic license plate detection method that is able to detect the license plates automatically with an appropriate accuracy, when the images are shadowed. Therefore, in this part, the details of the proposed method are addressed based on Figure 3.

\section{A. Converting the Color Image to the Gray Image}

A color image is a combination of three components of the color image: Red (R), Green (G) and Blue (B) with sizes of $M \times N$. The proposed method receives the color image as the input. In most cases, a license plate includes white characters in black background or black characters in white background. Some of the states-owned or diplomatic vehicles have license plates with black characters in green or yellow backgrounds [25]. This color diversity, however, is of no importance in the proposed method since no color information is used for the license plate detection from the image. Color information is very sensitive to non-uniform light and therefore, causes difficulties for detecting the license plate from the image. In the proposed method, thus, the gray image (binary) is used at each stage. Furthermore, compared to the color image, processing the gray image is easier and occupies less space. Relation 1 is used to convert the color image to the gray one [24]:

$$
\operatorname{Gray}(i, j)=0.30 * R(i, j)+0.59 * G(i, j)+0.11 * B(i, j)
$$

\section{B. Contrast Enhancement and Noise Removal}

\section{1) Removing the Shadows and Contrast Improvement}

As we know, probabilities indicate the chances for an event to take place. Probability is usually used for describing the subjective (mental) perspective of the statements which cannot be absolutely considered as true [26]. The concerned statements are of the types that state "Will a particular event take place?" our perspective is of the type that states "How sure are we about the occurrence of such event?" The extent of our confidence can be numerically expressed, and such value is a number ranging from 0 to 1 which is called probability [27]. The more probable an event is, the more confident we are that such event will take place. In fact, probability is the extent of our confidence about a (random) event to take place. On the other hand, we know that cumulative distribution is a part of a given sequence. Now, to remove the shadows from the image, probabilities can be used. For this purpose, first the histogram of the image should be used to obtain the existing pixel dispersion. Then the probability of each pixel is calculated and its cumulative distribution in the gray image is obtained. After inserting the obtained number in the image, we will be able to improve the contrast and remove the shadows from it as well. Figure 4 displays a sample of a shadowed image with low ambient light along with its relative histogram. Figure 5 displays the same Figure 4, after applying shadow removal algorithm and contrast improvement. As it is evident in the histogram of this image, after applying the proposed method, the ambient light is improved and shadows are removed from the image. In Figure 4, accumulation of pixels is on the right side of the chart ( 0 to 100) in the dark area and after applying the proposed technique, the pixels are distributed in the chart and 
the accumulation is on the right side of the chart and in the light area. This method can be used for color images as well. To do that, it is necessary to apply this method to each dimension of the image. For instance, if we assume an image with the dimensions of $M \times N$, we can calculate the probability of each pixel and the cumulative distribution, using Relations 2 and 3. Figure 6 displays a sample of the proposed shadow removal method.

$$
\begin{gathered}
P\left(z_{k}\right)=\frac{n_{k}}{M \times N} \\
E q\left(z_{i}\right)=\sum_{i=0}^{i=k} P\left(z_{i}\right) \times 256
\end{gathered}
$$

Where $M$ and $N$ are the image dimensions, $n_{k}$ is the number of times that $z_{k}$ light intensity has occurred in the image, and $\mathrm{P}\left(z_{k}\right)$ is the occurrence probability of $z_{k}$, and $E q\left(z_{i}\right)$ is the cumulative distribution.

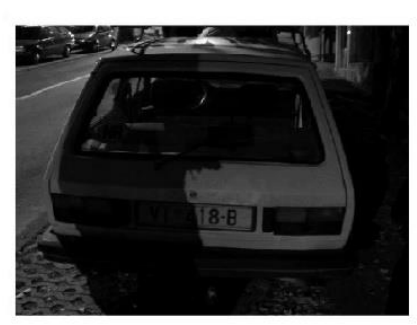

(a)

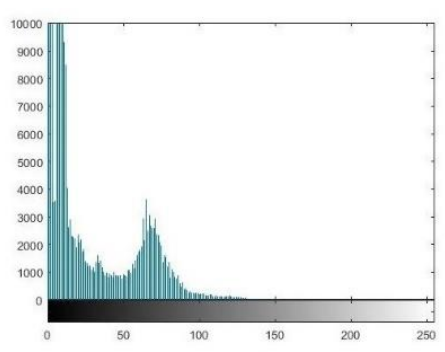

(b)

Fig. 4. (a) Shaded gray image in low light region, (b) Image's histogram

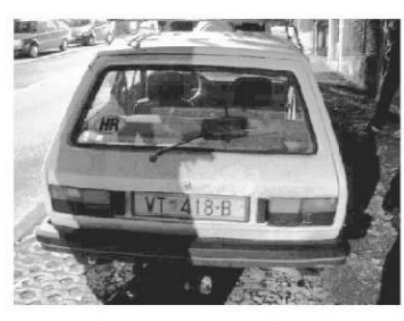

(a)

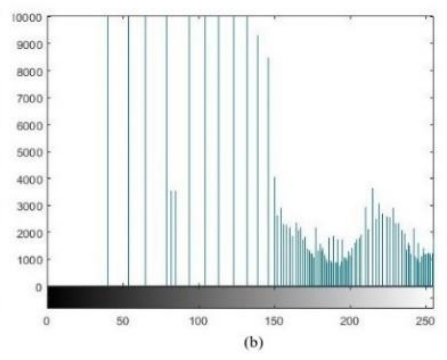

Fig. 5. (a) Output image after noise removal algorithm and enhanced contrast, (b) Image's histogram
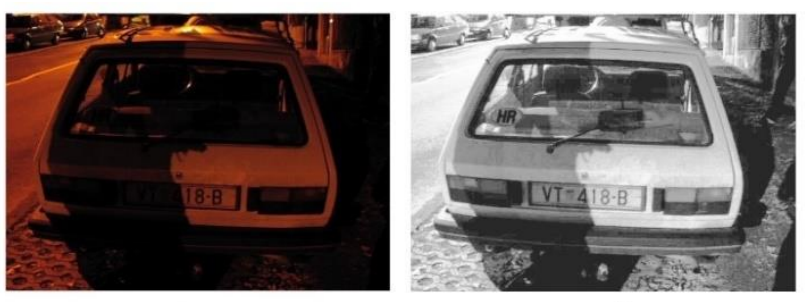

Fig. 6. The proposed shadow removal process, (a) Input image, (b) Gray scale image after shadow removal

\section{2) Tamura's Contrast Improvement}

The input image, due to different conditions such as night shot, blurred and shadowed images, has a high potential for low image contrast. The vertical and horizontal lines in the image cannot be easily detected in the images shot in the night, due to low ambient light and the effects of the camera's flash and shaking, which in turn affect the performance of the proposed license plate detection method in the next stages. Furthermore, the existence of shadows in the image leads to short sight or low contrast in the image. Here, we consider the shadow removal issue as contrast improvement at this stage. In order to detect the license plate area in the image successfully, we need to improve the noise-removed image. However, contrast improvement, without limitation or having the amount of current contrast of the image, leads to new problems such as increased noise, increased number of candidate areas as license plates and increased areas as license plate borders. For this purpose, a new method is applied that utilizes Tamura's contrast jointly with limited contrast of adaptive histogram equalization [15]. The value of Tamura's contrast gives us the contrast of the image within the range of 0 to 1 , and to control the range of contrast in the limited contrast of adaptive histogram equalization, we use Formula 5, for which the Tamura's value is deducted from 1 and then is divided to a constant number. Here, the constant number is the divisor that easily controls the value of Climit. Empirically, the value of this constant number is assumed equal to 200, by which Climit will be a value between 0 and 0.005 . Formula 5 displays the procedure for obtaining Climit [15].

$$
A=\frac{\mu_{4}}{\sigma^{4}} \quad, \quad F_{c o n}=\frac{\sigma}{A^{n}}
$$

Where $F_{c o n}$ is Tamura's contrast value, $\sigma$ is the standard deviation of image brightness, $\mu_{4}$ is the fourth torque around the mean and $n=0.25$. The output contrast value of this equilibrium is a number between 0 and 1 . A high value of Tamura's contrast indicates a high contrast and a low value of Tamura's contrast indicates a low contrast of the image.

$$
C_{\text {limit }}=\frac{\operatorname{abs}\left(\frac{\left(1-F_{\text {con }}\right)}{d i v}\right)}{255}
$$

In the above formula, $d i v=200$ and $F_{c o n}$ is obtained from Formula 4. This formula generates the contrast range in a way that if an image has a low contrast, it increases the contrast range and if an image has a high contrast, it decreases the contrast range. When we use the contrast range in the adaptive histogram equalization, it causes effective improved contrast in a noise-removed image and obtains an improved image as well.

\section{Opening Operation}

Before binarizing the image, we should remove small white particles, unnecessary license plate candidate border areas and narrow connections between large white objects. For this purpose, we use opening morphological operation (erosion and dilation), utilizing the elemental rectangular structure. The size of the rectangle depends on the size of the license plate area. Here, the size of the rectangle is assumed as 
$3 \times 35$. In the next step, the image derived from the morphological operation is deducted from the gray image.

\section{Binarizing the Image}

In a gray image, license plate areas have a considerable disorder due to the brightness intensity of the license plate characters and the background. This means that the occurrence of the brightness intensity change among the pixels is high in the license plate area, considering the contrast between the texture and the background. Here, we use hysteresis thresholding in order to binarize the images [29]. After applying opening morphological operation to the image, the image is binarized using Formula 6 . In this formula, $T h_{\text {low }}=0.1$ and $T h_{\text {high }}=0.3$.

$$
I_{t}(x, y)= \begin{cases}\text { foreground (strong) } & \text { if } I_{t}(x, y)>T h_{\text {high }} \\ \text { background } & \text { if } I_{t}(x, y)<T h_{\text {low }} \\ \text { candidate }(\text { week }) & \text { otherwise }\end{cases}
$$

Any pixel with a value lower than $T h_{l o w}$ is considered as a background and any pixel with a value higher than $T h_{h i g h}$ is considered as a strong background.

\section{E. Opening operation Followed by Closing Operation}

In this stage, the erosion and dilation morphological operations are once again applied in order to remove the small holes and expand the white areas. First, an opening operation is applied to the image (erosion and then dilation) and subsequently, a closing operation (dilation and then erosion) is applied. A similar elemental structure (rectangular shaped) is used in both of these operations with different sizes. The size in the opening operation is assumed as $3 \times 3$ and the size in the closing operation is assumed as $3 \times 19$ for the element generation.

\section{3) Noise Removal}

The proposed automatic license plate detection method uses local disorders (light intensity transfers) of an image as the initial feature for locating the license plate, since disorders in the area of license plate is high due to the text content. However, existence of the noise (such as small particles of dirt, dust and so on) leads to increased unnecessary disorders in the image and thus, leads to an increased number of candidate areas for license plates. In order to remove small noises, therefore, Wiener's local noise removal filter with the window size of $3 \times 3$ is used [28]. Using Wiener's local noise removal filter at this stage assists us to deal with a fewer number of unnecessary elements in the background in the next stage for automatic license plate detection. Figure 7 displays a sample of such noise removal. As it is evident in the image, Wiener's filter has removed small noises without any damage to the details of the image, and this is one of the significant advantages of this filter.

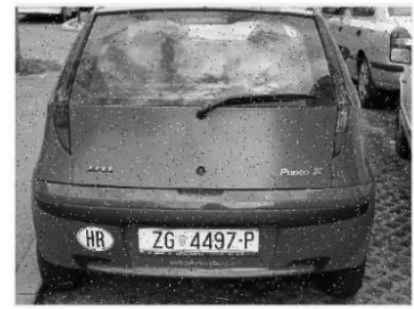

(a)

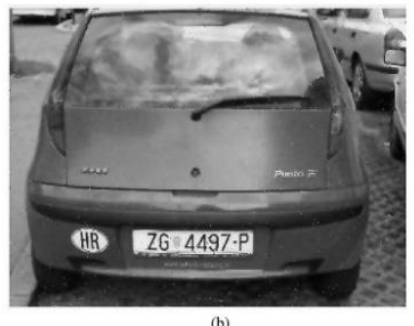

(b)
Fig. 7. (a) A noise-induced gray scale image, (b) The image after using Wiener noise filter.

\section{F. Connected Component Analysis and Filtering Non-LP Regions}

\section{1) Analysis of the Connected Components}

All of the connected components in the image obtained from the morphological operations are labeled in this step based on eight neighborhood connections. Figure 8 demonstrates a sample gray image, improved image, the image obtained from morphological operation, and the image with connected components along with their labels. Among these areas, only one is the target license plate area and the others are not. A filtering or a good analysis is required in the next step in order to locate the target license plate area.

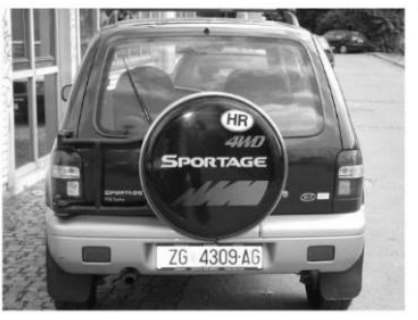

(a)

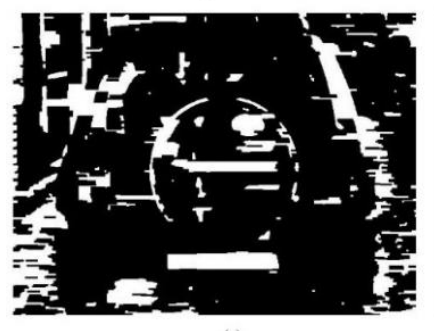

(c)

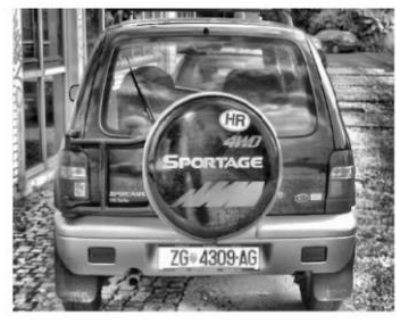

(b)

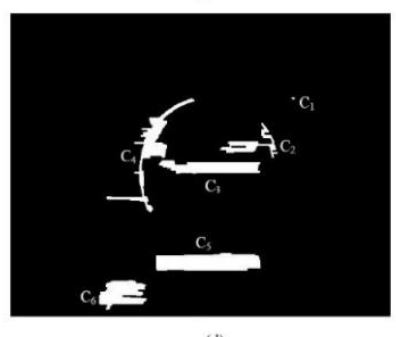

(d)
Fig. 8. The process of labeling the connected components, (a) Gray scale image, (b) Enhanced image, (c) Output image from morphological operation stage, (d) The image of the connected component based on 8 neighboring connections.

\section{2) Filtering non- License Plate Areas}

After analyzing the connected components, we have a number of license plate candidate areas. Among these areas, however, only one is our target license plate candidate. In the last stage, we filter all non-license plate areas from the candidate areas based on a number of main features such as size, surface area and entropy. For this purpose, the proposed automatic license plate detection method applies the following two steps to the images:

a) Filtering Non-License Plate Areas based on Size and Surface Area 
At first, the non-license plates are filtered based on the resolution or the size of the license plate in the image. The text area of the license plates of different vehicles is almost the same in the images that are taken from a specific distance, since license plates usually obey a particular standard while being produced. The images used for this research (with a resolution of $640 \times 480$ ) were shot almost within a distance of 1 to 3 meters; and under this condition, the mean resolution of the unfiltered areas of the license plate is about $40 \times 120$. Under this condition, the least values for the length and width of the license plate consist of 60 and 30 pixels, respectively. Therefore, any license plate with a length and width of less than 60 and 30 pixels will be rejected. To filter the non-license plate areas, we use the proportion of length to width (surface area) of the candidate areas as a feature. Almost in all license plates, the horizontal dimension is longer than the vertical one. Even in lopsided license plates, the vertical dimension is no longer. We have empirically found out that those candidate license plate areas, in which the proportion of the length to the width is less than 0.15 , are not actually the target license plate areas. Therefore, such candidate license plate areas will be rejected as non-license plates. Figure 9 demonstrates a sample of the images of candidate license plate areas that are rejected based on the surface area and size.

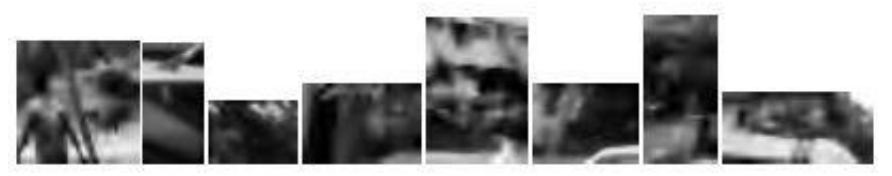

Fig. 9. Examples of candidate LP images which are rejected as non-LP images due to undersized resolution.

\section{b) Filtering non-License Plate Areas based on Entropy}

Here, we will use a relatively new method based on measuring the entropy (the amount of information) in order to filter the non-license plate images. Entropy means measuring the average number of the required bits for an image display [15]. Due to the existence of the numbers and words on a license plate (often black texts in a white background), the extent of the dispersion of black and white pixels are higher in such areas. Therefore, before calculating the entropy, a local vertical counter filter with the size of $3 \times 1$ is applied to the perimeter of the binary image. Then the perimeter binary image is binarized based on the value of the vertical counting. If the local counting value is 3 , then number 1 is assumed and otherwise 0 is assumed. We have assumed entropy as the amount of information in bit per pixel unit at the time of filtering. The highest value of entropy is 1 for binary images. We have empirically found that if the value of entropy is higher than 0.15 , then the candidate area is not a license plate and thus, it will be considered as non-license plate area.

In this part, we have addressed and described a new automatic license plate detection method that is capable of solving the issue of shadowed images. Here, the performance of the proposed automatic license plate detection method is evaluated and analyzed using various image data bases. Furthermore, the proposed automatic license plate detection method will be compared with two other new automatic license plate detection methods.

\section{RESULT}

We have tested our proposed method on 1000 images from three databases of University of Zagreb [15], Numberplates.com [30] and National Technical University of Athens [16]. These images included different environmental conditions such as shadowed images, low contrast images and night and day shot images. The samples of license plates detected by the proposed automatic license plate method are provided in Figure 10. Also, Table 1 presents the rate of the license plate detection under different environmental conditions utilizing the proposed automatic license plate detection method.
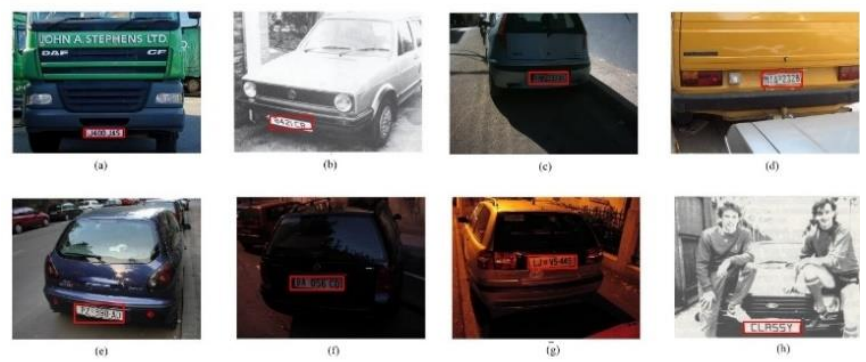

Fig. 10. Examples of license plate detection by proposed automatic license plate detection in different conditions: (a), (d), (e) day light, (b), (h) images with low contrast, (c) shaded image, (f), (g) night shot images.

As it is evident in Table 1, the proposed method has a performance of 97.30 percent in detecting license plates in shadowed images. This is due to the fact that the proposed method uses probabilities and cumulative distribution in the shadow removal and contrast improvement stage and therefore, is able to remove the existing shadow from the image and improve the amount of light in the image (increased brightness). Furthermore, the proposed method is able to reach an accuracy of 76.50 percent in images with low contrast because it uses Tamura's contrast improvement. The reason is that such images face problems in identifying and detecting the license plates due to sharp edges between the text and the background of license plates. Also, before filtering nonlicense plate areas, candidate areas include all target license plates. However, during the course of filtering, choosing a wrong target license plate leads to a lower rate of license plate detection. In addition, the proposed method has a good performance for the night shot and day shot images, which proves that this method is not exclusive to shadowed images.

TABLE I. PERFORMANCE OF THE PROPOSED AUTOMATIC LiCENSE Plate Detection MeThod in DifFERENT Conditions: SHADEd IMAGES, IMAGES WITH LOW CONTRAST, AND CAPTURED IMAGES IN DAY AND NIGHT LIGHT

\begin{tabular}{|l|l|l|l|}
\hline \multirow{2}{*}{ Condition } & \multicolumn{3}{|l|}{$\begin{array}{l}\text { Performance of the proposed automatic } \\
\text { license plate detection method in } \\
\text { different conditions }\end{array}$} \\
\cline { 2 - 4 } & $\begin{array}{l}\text { Number } \\
\text { of } \\
\text { Images }\end{array}$ & $\begin{array}{l}\text { Number of } \\
\text { Detected } \\
\text { Images }\end{array}$ & $\begin{array}{l}\text { Detection } \\
\text { Rate in } \\
\text { Percent }\end{array}$ \\
\hline Shaded Images & 127 & 124 & $97 / 30$ \\
\hline Images with Low Contrast & 76 & 58 & $76 / 50$ \\
\hline Captured Images in Night & 166 & 148 & $98 / 20$ \\
\hline
\end{tabular}




\begin{tabular}{|l|l|l|l|} 
Captured Images in Day & 631 & 622 & $98 / 50$ \\
\hline
\end{tabular}

The proposed automatic license plate detection method is evaluated and compared with two other license plate detection methods $[14,15]$. These two methods are deployed using MATLABR2015b software and tested on available databases including 1000 images under different environmental conditions. The results obtained from the evaluation of the images from the database of University of Zagreb are summarized in Table 2, the results of Numberplates.com are summarized in Table 3 and the results of National Technical University of Athens are summarized in Table 4.

TABLE II. EVALUATING AND COMPARING THE PERFORMANCE OF Methods $[14,15]$ With THE PRoposed Automatic LicENSE Plate DETECTION ON IMAGES OBTAINED FROM THE DATABASE OF THE UNIVERSITY OF ZAGREB.

\begin{tabular}{|l|l|l|l|}
\hline \multirow{2}{*}{$\begin{array}{l}\text { Automatic } \\
\text { License Plate } \\
\text { Detection }\end{array}$} & \multicolumn{3}{|l|}{$\begin{array}{l}\text { Evaluating and comparing the performance of } \\
\text { methods [14, 15] with the proposed automatic } \\
\text { license plate detection on images obtained from } \\
\text { the database of the University of Zagreb }\end{array}$} \\
\cline { 2 - 4 } & $\begin{array}{l}\text { Number of } \\
\text { Detected LP } \\
\text { from 515 } \\
\text { Images }\end{array}$ & $\begin{array}{l}\text { Detection } \\
\text { Rate in } \\
\text { Percent }\end{array}$ & $\begin{array}{l}\text { Average } \\
\text { Running Time } \\
\text { in Seconds }\end{array}$ \\
\hline Method [14] & 370 & $71 / 80$ & $2 / 33$ \\
\hline Method [15] & 112 & $21 / 70$ & $0 / 83$ \\
\hline Proposed Method & 466 & $30 / 90$ & $0 / 39$ \\
\hline
\end{tabular}

TABLE III. EVAluating AND COMPARING THE PERFORMANCE OF Methods $[14,15]$ With the Proposed Automatic LiCENSE Plate DETECTION ON IMAGES OBTAINED FROM THE DATABASE OF THE NUMBERPLATES.COM.

\begin{tabular}{|l|l|l|l|}
\hline \multirow{2}{*}{$\begin{array}{l}\text { Automatic } \\
\text { License Plate } \\
\text { Detection }\end{array}$} & \multicolumn{3}{|l|}{$\begin{array}{l}\text { Evaluating and comparing the performance of } \\
\text { methods [14, 15] with the proposed automatic } \\
\text { license plate detection on images obtained from } \\
\text { the database of the NumberPlates.com }\end{array}$} \\
\cline { 2 - 4 } & $\begin{array}{l}\text { Number of } \\
\text { Detected LP } \\
\text { from 330 Images }\end{array}$ & $\begin{array}{l}\text { Detection } \\
\text { Rate in } \\
\text { Percent }\end{array}$ & $\begin{array}{l}\text { Average } \\
\text { Running } \\
\text { Time in } \\
\text { Seconds }\end{array}$ \\
\hline Method [14] & 211 & $63 / 93$ & $1 / 48$ \\
\hline Method [15] & 94 & $28 / 48$ & $2 / 21$ \\
\hline Proposed Method & 323 & $90 / 30$ & $0 / 44$ \\
\hline
\end{tabular}

TABLE IV. EVAluating AND COMPARING THE PERFoRMANCE OF METHODS $[14,15]$ wITH THE PROPOSED AUTOMATIC LICENSE Plate DETECTION IN IMAGES OBTAINED FROM THE DATABASE OF THE NATIONAL TECHNICAL UNIVERSITY OF ATHENS.

\begin{tabular}{|l|l|l|l|}
\hline \multirow{2}{*}{$\begin{array}{l}\text { Automatic } \\
\text { License Plate } \\
\text { Detection }\end{array}$} & \multicolumn{2}{|l|}{$\begin{array}{l}\text { Evaluating and comparing the performance of } \\
\text { methods [14, 15] with the proposed automatic } \\
\text { license plate detection in images obtained from the } \\
\text { database of the National Technical University of } \\
\text { Athens }\end{array}$} \\
\cline { 2 - 4 } & $\begin{array}{l}\text { Number of } \\
\text { Detected LP } \\
\text { from 155 } \\
\text { Images }\end{array}$ & $\begin{array}{l}\text { Detection } \\
\text { Rate in } \\
\text { Percent }\end{array}$ & $\begin{array}{l}\text { Average } \\
\text { Running Time } \\
\text { in Seconds }\end{array}$ \\
\hline Method [14] & 96 & $61 / 93$ & $1 / 13$ \\
\hline Method [15] & 23 & $14 / 83$ & $1 / 56$ \\
\hline
\end{tabular}

\begin{tabular}{|l|l|l|l} 
Proposed Method & 153 & $98 / 70$ & $0 / 88$
\end{tabular}

The shadows are removed from the images and the amount of light is adequately modified due to utilizing the histogram modification, cumulative distribution, probability of the pixels and Tamura's contrast improvement in the image preprocessing stage. This enables the proposed method to reach a higher accuracy than the two other compared methods. The two other compared methods have not presented any techniques for modifying the ambient light; also, to remove the shadows, they have used Bernsen algorithm which is very noise sensitive. All the license plates are extracted from the gathered 1000 images and are categorized in three classes based on the mean intensity of brightness of the license plates: The mean brightness intensity of 0 to 85 pixel (high brightness), 86 to 176 pixels (medium brightness) and 171 to 255 pixels (low brightness). Chart 1 demonstrates the evaluation and comparison of the methods [14, 15] with the proposed method in the above mentioned classes; as it is shown, the proposed method has a high accuracy not only for images with high brightness but also for those with low brightness and shadows.

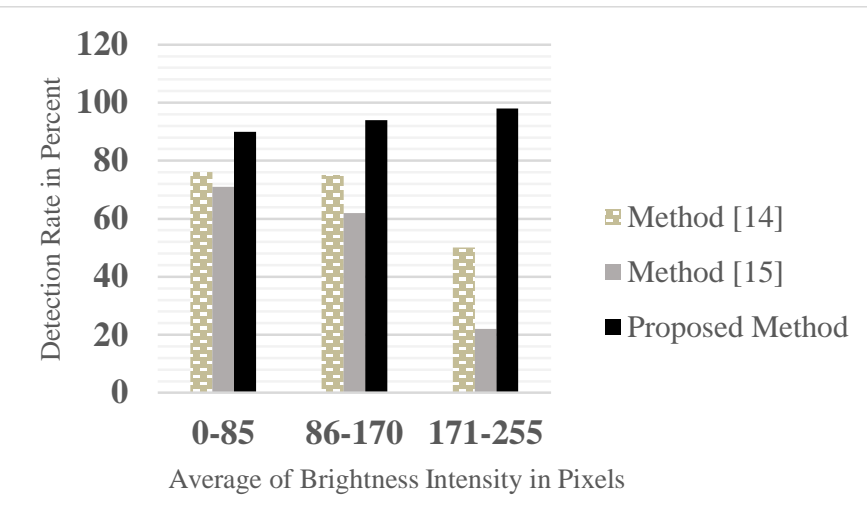

Chart. 1. Evaluating and comparing the performance of the mentioned methods with the proposed method based on the average brightness of plates.

Furthermore, all gathered images are categorized based on the distances of the camera from the license plate, which are 1, 3, 5 and 10 meters. Chart 2 demonstrates the evaluation and comparison of the methods $[14,15]$ with the proposed method in different distances and based on the categorization of the mean brightness intensity. As it is evident, all three methods had relatively good results within the distances of 1 to 3 meters. However, as the license plate distances from the camera and the existence of unwanted objects in the image increase, the accuracy of license plate detection reduces in all methods. As the distance increases, the size of the license plate varies and becomes smaller and therefore, we face lower accuracy of license plate detection in all methods. Furthermore, as the distance increases, the scope of the view of the image increases as well and therefore, more unwanted objects are observed in the image which in turn leads to an inaccurate detection of license plate area in all methods. 


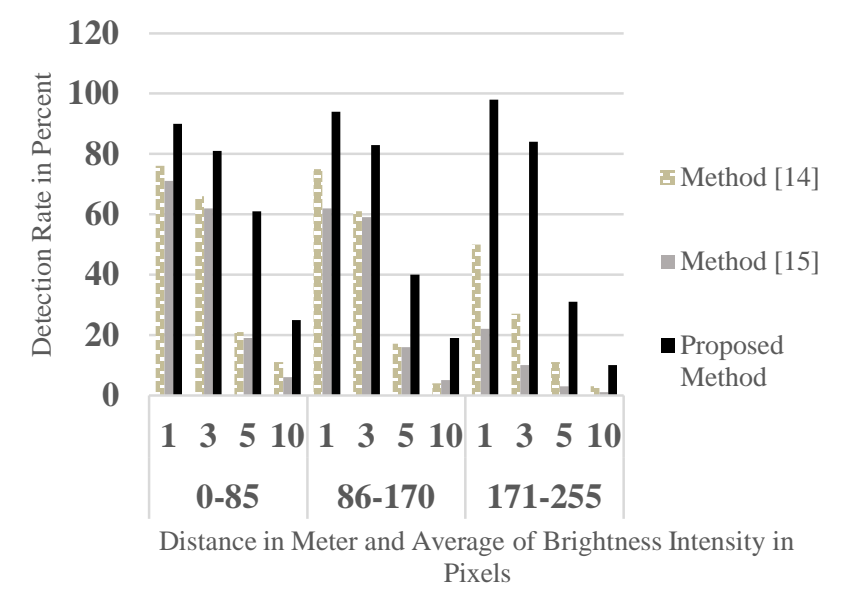

Chart. 2. Evaluating and comparing the mentioned methods with the proposed method at intervals of $1,3,5$ and 10 meters based on the average brightness of plates.

Compared with other available methods, the introduced shadow removal and contrast improvement method in this research showed a better performance in terms of identification and detection of the license plate. The proposed method, under the shadowed image condition as well as the regular condition, leads to an improved contrast of the image and therefore, results in an increased license plate detection rate. The proposed method was tested on 1000 images from three databases under different conditions and in total, achieved the mean detection rate of 95.62 percent. The results of English written license plates and the images with symbolic license plates are provided. The results prove that, compared to two other available automatic license plate detection methods, the proposed automatic license plate detection method shows a better performance; it also shows much shorter average performance time.

\section{CONCLUSION}

A new license plate detection method, consisting of six stages, was introduced in this research. In the stage of contrast improvement and shadow removal, the method of histogram equalization based on probability was utilized and therefore, the shadow was identified in the image and then removed, which led to an improved image contrast. The color image was converted into the gray image in the first stage. In the next stage, in order to improve the image contrast and identify and remove the shadows, a new method based on histogram equalization on the basis of probabilities was presented. In this stage and for the purpose of identifying and removing shadows, the histogram of the image was firstly calculated for each dimension and then the probability of existence of each pixel in the whole image was obtained. After calculating the cumulative sum of the pixels and replacing them in the image, we can remove shadows from the image and improve their resolution. The proposed method can also be used for removing the shadows and improving the resolution in color images. For this purpose, it is only necessary to apply the proposed method to all three dimensions of the image and then apply Wiener's noise removal filter to the gray image in order to remove small noises. In this stage, in order to solve the issue of the low contrast of images (for instance, night shots, blurred and shadowed images), Tamura's contrast improvement method is used. In the next stage, morphological opening operation is applied to the improved images and the obtained image is deducted from the gray image. Then the obtained image is binarized using hysteresis threshold and later, morphological operations are again applied in order to fill the holes and expand the white colored areas. In the next stage, all of the connected components (8 neighborhoods) are categorized. Such categorized components are actually the license plate candidate areas for the next stage. Eventually, in the last stage, non-license plate areas are rejected based on size and surface area. Furthermore, the filtering technique based on entropy is applied, in the end, in order to filter other non-license plate areas. After filtering, the remaining license plate candidate areas are assumed as the target license plate areas. The proposed automatic license plate detection method was tested on 1000 images from three different databases and in total, reflected a detection rate of 95.62 percent and an average performance time of 0.39 seconds. The proposed automatic license plate detection method has only considered the rectangular license plates and other shapes of license plates such as square license plates are not included. Also, in this method, no solutions are proposed to solve the issues concerning the horizontal or vertical deviations of the license plates. We attempt to solve such issues in the future. In addition, further researches should be done in this field in order to use the proposed method in everyday applicable programs.

\section{REFERENCES}

[1] Jiang, C. and M.O. Ward, Shadow identification, in Proceedings 1992 IEEE Computer Society Conference on Computer Vision and Pattern Recognition. 1992, IEEE: Champaign, USA. p. 606-612.

[2] El-Said, S.A., Shadow aware license plate recognition system. Soft Computing, 2015. 19(1): p. 225-235.

[3] Zhu, S., S. Dianat, and L.K. Mestha, End-to-end system of license plate localization and recognition. Journal of Electronic Imaging, 2015. 24(2): p. 023020-023020.

[4] Fu, R., The Research and Design of Vehicle License Plate Recognition System in Traffic Management System. International Journal of Signal Processing, Image Processing and Pattern Recognition, 2016. 9(3): p. 445-456.

[5] Zang, D., et al., Vehicle license plate recognition using visual attention model and deep learning. Journal of Electronic Imaging, 2015. 24(3): p. 033001-033011.

[6] Hsieh, J.-W., S.-H. Yu, and Y.-S. Chen, Morphology-based license plate detection in images of differently illuminated and oriented cars. Journal of electronic Imaging, 2002. 11(4): p. 507-516.

[7] Tadic, V., M. Popovic, and P. Odry, Fuzzified Gabor filter for license plate detection. Engineering Applications of Artificial Intelligence, 2016. 48(5): p. 40-58.

[8] Tian, J., et al., Chinese license plate character segmentation using multiscale template matching. Journal of Electronic Imaging, 2016. 25(5): p. 053005-053005.

[9] Dehshibi, M. and R. Allahverdi, Persian Vehicle License Plate Recognition Using Multiclass Adaboost. International Journal of Computer and Electrical Engineering, 2012. 4(3): p. 355-358.

[10] Chai, H.Y., et al., Elimination of character-resembling anomalies within a detected region using density-dependent reference point construction in an automated license plate recognition system. Journal of Electronic Imaging, 2016. 25(6): p. 061614-061614.

[11] Dey, S., A. Choudhury, and J. Mukherjee, An Efficient Technique to Locate Number Plate using Morphological Edge Detection and 
Character Matching Algorithm. International Journal of Computer Applications, 2014. 101(15): p. 36-41.

[12] Postolache, A. and J. Trécat, Pyramidal approach to license plate segmentation. Journal of Electronic Imaging, 1996. 5(3): p. 402-409.

[13] Dastjerdi, H.V., V. Rostami, and F. Kheiri, Automatic license plate detection system based on the point weighting and template matching, in 7th Conference on Information and Knowledge Technology (IKT). 2015, IEEE: Urmia, Iran. p. 1-5.

[14] Hommos, O., et al., HD Qatari ANPR system, in International Conference on Industrial Informatics and Computer Systems (CIICS 2016). 2016, IEEE: Sharjah, UAE. p. 1-5.

[15] Azam, S. and M.M. Islam, Automatic license plate detection in hazardous condition. Journal of Visual Communication and Image Representation, 2016. 36(3): p. 172-186.

[16] Anagnostopoulos, C.N.E., et al., A license plate-recognition algorithm for intelligent transportation system applications. IEEE Transactions on Intelligent transportation systems, 2006. 7(3): p. 377-392.

[17] Deb, K., H.-U. Chae, and K.-H. Jo, Vehicle license plate detection method based on sliding concentric windows and histogram. Journal of computers, 2009. 4(8): p. 771-777.

[18] Wang, Y.-R., W.-H. Lin, and S.-J. Horng, A sliding window technique for efficient license plate localization based on discrete wavelet transform. Expert Systems with Applications, 2011. 38(4): p. 31423146.

[19] Ghosh, A.K., et al., Automatic license plate recognition (ALPR) for Bangladeshi vehicles. Global Journal of Computer Science and Technology, 2011. 11(21): p. 69-73.

[20] Joarder, M.M.A., et al., Bangla automatic number plate recognition system using artificial neural network. Asian Transactions on Science \& Technology (ATST), 2012. 2(1): p. 1-10.

[21] Hsu, G.-S., J.-C. Chen, and Y.-Z. Chung, Application-oriented license plate recognition. IEEE transactions on vehicular technology, 2013.
62(2): p. 552-561.

[22] Nejati, M., A. Majidi, and M. Jalalat, License plate recognition based on edge histogram analysis and classifier ensemble, in Signal Processing and Intelligent Systems Conference (SPIS 2015). 2015, IEEE: Tehran, Iran. p. 48-52.

[23] Li, H. and C. Shen, Reading Car License Plates Using Deep Convolutional Neural Networks and LSTMs. arXiv preprint arXiv:1601.05610, 2016. 3(8): p. 1-17.

[24] Kasaei, S.H., S.M. Kasaei, and S.A. Kasaei, New Morphology-Based Method for RobustIranian Car Plate Detection and Recognition. International Journal of Computer Theory and Engineering, 2010. 2(2): p. 264.

[25] Rabee, A. and I. Barhumi, License plate detection and recognition in complex scenes using mathematical morphology and support vector machines, in The 21st International Conference on Systems, Signals and Image Processing (IWSSIP 2014). 2014, IEEE: Dubrovnik, Croatia. p. 59-62.

[26] Stuart, A. and J.K. Ord, Kendall's Advanced Theory of Statistics, Volume 1: Distribution Theory, Edward Arnold. 6 ed. Kendall's library of statistics. Vol. 1. 1994, London, UK: London : Edward Arnold ; New York : Halsted Press. 700.

[27] Feller, W., An introduction to probability theory and its applications. 3 ed. Probability and Statistics - Applied Probability and Statistics Section Series. Vol. 1. 1968, New Jersey, USA: John Wiley \& Sons LondonNew York-Sydney-Toronto. 528.

[28] Benesty, J., et al., Study of the Wiener filter for noise reduction, in Speech Enhancement. 2005, Springer. p. 9-41.

[29] Al Najjar, M., M. Ghantous, and M. Bayoumi, Hysteresis Thresholding, in Video Surveillance for Sensor Platforms. 2014, Springer New York: NY, USA. p. 147-174.

[30] Number Plates, Car registrations and DVLA plates - PRICE REDUCTIONS! 2017; Available from: http://www.numberplates.com/. 